\title{
Tocilizumab and COVID-19
}

\author{
Dhruva Chaudhry ${ }^{1}$, Pawan K Singh ${ }^{2}$
}

\begin{abstract}
The number of cases in COVID-19 pandemic is rising rapidly. There has not been a single effective proven medication for COVID-19 disease. Highest mortality has been reported among subjects who develop acute respiratory disease (ARDS). The histopathological analysis of lung specimens has given rise to theories that propose the major role of cytokine release syndrome in the development of ARDS. IL- 6 has often been found to be raised in subjects having severe disease. Tocilizumab is a selective inhibitor of the IL- 6 pathway and has been approved for various rheumatological diseases. Its use in COVID-19 has been evaluated following the success of other immunosuppressive drugs like steroids. The data in support of against its use in COVID19 are lacking. Similarly, the risk of early- and late-onset infections after tocilizumab in COVID-19 remains unknown. The study by Nasa et al. is a valuable addition to the evidence concerning its use. Despite multiple articles, its safety and efficacy in COVID-19 remain unknown. Caution must be used about its timing and role of IL-6 levels for disease monitoring.

Keywords: COVID-19, Cytokine release syndrome, Tocilizumab.

Indian Journal of Critical Care Medicine (2020): 10.5005/jp-journals-10071-23608
\end{abstract}

\section{INTRODUCTION}

COVID-19 pandemic has affected more than 25 million people and caused 847,602 deaths so far. ${ }^{1}$ There is no single antiviral drug or vaccine with proven efficacy to treat or prevent human coronavirus (SARS-CoV-2) infection.

Acute respiratory distress syndrome (ARDS) is the most severe and fatal complication of the COVID-19 disease. Inflammation and cytokine storm have been implicated as the principal responsible etiologies for pulmonary involvement in COVID-19 disease. ${ }^{2}$

Cytokine release syndrome (CRS), in severe form, is a lifethreatening acute systemic inflammatory syndrome, which is characterized by multiorgan failure and fever. It plays the central role in the pathogenesis of SARS-CoV-2 infection. In COVID-19 severe disease, it was observed that increased level of cytokines and especially interleukin-6 (IL-6) was a key factor of inflammation. ${ }^{3}$ The emerging role of immunosuppressive drugs like steroids and their potential mortality benefits have incited the curiosity of the role of targeted cytokine inhibitor therapy, like tocilizumab.

Tocilizumab is a recombinant humanized monoclonal antibody that binds to both membrane-bound as well as soluble forms of the IL- 6 receptor. Initially, tocilizumab was identified and used for multiple rheumatological diseases and in chimeric antigen receptor T (CAR T) cell therapies. ${ }^{4}$

\section{Tocilizumab Efficacy in COVID-19}

As tocilizumab is widely used in the treatment of IL-6-induced CRS, it is being tried as an off-label treatment for moderate to severe COVID-19 disease. The safety and efficacy of the use of tocilizumab in the treatment of COVID-19 patients are not yet proven.

Due to rapidly evolving evidence surrounding COVID-19, there are not much data over the use of tocilizumab in COVID-19. Neither safety nor efficacy has been prospectively tested in well-designed trials. But few centers have reported the use of tocilizumab in COVID-19 patients and its effectiveness. Toniati et al. reported 100 subjects from Italy involving the use of tocilizumab. They were able to demonstrate that tocilizumab was able to reduce the Brescia COVID respiratory severity score (BCRSS) significantly and that too in a shorter period. But being a single center and having no
1,2 Department of Pulmonary and Critical Care Medicine, Pandit Bhagwat Dayal Sharma Postgraduate Institute of Medical Sciences, Rohtak, Haryana, India

Corresponding Author: Pawan K Singh, Department of Pulmonary and Critical Care Medicine, Pandit Bhagwat Dayal Sharma Postgraduate Institute of Medical Sciences, Rohtak, Haryana, India, Phone: +91 8437013094, e-mail: ga.ps.complete@gmail.com

How to cite this article: Chaudhry D, Singh PK. Tocilizumab and COVID-19. Indian J Crit Care Med 2020;24(9):741-743.

Source of support: Nil

Conflict of interest: None

control arms were the main lacunas of this index study. ${ }^{5}$ Similarly, in a retrospective study by Guaraldi et al., it was found that tocilizumab was associated with significantly reduced deaths as well as the requirement of invasive mechanical ventilation. ${ }^{6}$ Price et al., in their prospective observational study of 239 subjects, also supported the role of tocilizumab in improving outcomes of severe COVID-19 cases. They found that algorithm that included the use of tocilizumab for targeting CRS influenced use of mechanical ventilation and survival in these cases. ${ }^{7}$ But despite these studies, in a systematic review conducted by Cortegiani et al., no conclusive evidence of safety or efficacy could be derived due to methodological flaws and observational nature of the included studies. ${ }^{8}$

Recently, preliminary results of COVACTA trials results were released. Despite being a World Health Organization (WHO)approved double-blinded, randomized controlled trial and having a broad eligibility list, tocilizumab failed to improve clinical status or mortality against the standard of care control. The subgroup analysis and detailed results are still awaited. ${ }^{9}$

The study of Nasa et al. is a valuable addition to the literature. Data of 85 subjects of COVID-19 disease requiring ICU care were included in the study. They found that the use of tocilizumab was associated with lower mortality (9.1\% vs $57.1 \%, p=0.001)$ and lower need for escalation of therapy $(22.7 \%$ vs $65.1 \%, p=0.001)$. 
Similarly, biomarkers of inflammation and CRS were also less in the tocilizumab group as compared to the standard of care group. These findings are seen as proof of the role of tocilizumab therapy in COVID-19 disease.

Given that IL- 6 plays a key driver role in the inflammatory storm in the patients of COVID-19, various authors have proposed simultaneous use of antiviral and anti-inflammatory treatment.

Criteria for the use of tocilizumab in COVID-19 have been varying but predominantly include the following:

- Extensive and bilateral lung disease in severely ill patients with elevated IL-6 levels

- Alternatively, a high level of D-dimer and/or C-reactive protein (CRP) and/or ferritin and/or progressively increasing fibrinogen

- Worsening of respiratory exchanges such as to require noninvasive or invasive support of ventilation

Tocilizumab should be used in a selective group of patients. Timing to administer the drug is very crucial. However, evidence regarding the proper timing of administration is also not definitive. Based on current data, tocilizumab should be used in patients with significant lung involvement, moderately or severely ill patients with elevated IL- 6 level. If no clinical improvement has been observed, then one another dose can be used. Though lower doses of tocilizumab have been tried in various studies, their efficacy in severe disease is of limited value.

The subcutaneous route of administration of tocilizumab in COVID-19 has also been explored in anecdotal reports. As the subcutaneous route is already approved for rheumatoid arthritis (RA), it is presumed that same can be applied here as well. A retrospective analysis by Potere et al. shows that it is feasible to give tocilizumab via the subcutaneous route, and also it leads to significant fall in CRP values and requirement of oxygen supplementation when compared to the standard of care group. ${ }^{10}$ But its efficacy and safety against intravenous administration remain unknown.

\section{Tocilizumab Safety and Potential for Toxicity}

Initially, the adverse reactions of tocilizumab for chronic administration were assessed in rheumatological disease only. In a double-blinded, randomized study on 623 patients with RA who were given tocilizumab vs placebo, it was found that serious infections are more prevalent with the tocilizumab. ${ }^{4} \mathrm{~A}$ metaanalysis with 2,188 patient-years exposure to look for efficacy and safety of tocilizumab alone in the treatment of moderate to severe RA, ${ }^{11}$ it was found that serious adverse events like infections along with mild abnormality in the lipid profile or the liver function test were common. The most common side effect of tocilizumab is an increase in serum cholesterol, AST, ALT, and injection site reaction. Safety and toxicity of tocilizumab in other diseases help in making potential exclusion criteria in COVID-19 treatment. Among infections, reactivation of latent tuberculosis is a common threat after administration of tocilizumab.

For safety assessment in a study by Pettit et al., it was found that on long-term follow-up of recipients of tocilizumab in COVID19 severe disease, late-onset infections were more common. And a higher number of cases did have tocilizumab-related complications like deranged liver function tests and infusion-related allergenic reactions. ${ }^{12}$
Besides, it is worthwhile to mention that IL6 is not the appropriate marker of disease improvement as the soluble IL- 6 receptor immune complex can remain in the serum for the long time because of its longer half-life, even after tocilizumab administration. Also, IL- 6 levels can increase after tocilizumab administration because of less consumption of IL- 6 . $^{13}$

Apart from the apparent safety and efficacy endpoints, the role of drug interaction of the tocilizumab should be considered. Tocilizumab is a cytochrome P450 enzyme inducer and may decrease the serum concentration of cytochrome P450 3A4 substrates. It is recommended not to use rivaroxaban and apixaban in patients who are receiving tocilizumab. The dose of warfarin should also be modified with tocilizumab. Taken together, according to the data available, tocilizumab is safe in patients with severe or critically ill COVID-19 with the standard dose.

In conclusion, tocilizumab has been upcoming as a drug to watch, but it is associated with its own share of adverse effects. Use and recommendations for tocilizumab in COVID-19 moderate/ severe disease should be taken with caution as not only the supportive data are lacking but also long-term outcomes of tocilizumab in COVID-19 diseases are yet to be known.

\section{References}

1. Organization WH. Situation Report: WHO; 2020 [Available from: https://www.who.int/emergencies/diseases/novel-coronavirus-2019/ situation-reports.

2. Gibson PG, Qin L, Puah SH. COVID-19 acute respiratory distress syndrome (ARDS): clinical features and differences from typical preCOVID-19 ARDS. Med J Australia 2020;213(2):54-6.e1. DOI: 10.5694/ mja2.50674.

3. Wang J, Jiang M, Chen X, Montaner LJ. Cytokine storm and leukocyte changes in mild versus severe SARS-CoV-2 infection: review of 3939 COVID-19 patients in China and emerging pathogenesis and therapy concepts. J Leukoc Biol 2020;108(1):17-41. DOI: 10.1002/ JLB.3COVR0520-272R.

4. Scott LJ. Tocilizumab: a review in rheumatoid arthritis. Drugs 2017;77(17):1865-1879. DOI: 10.1007/s40265-017-0829-7.

5. Toniati P, Piva S, Cattalini M, Garrafa E, Regola F, Castelli F, et al. Tocilizumab for the treatment of severe COVID-19 pneumonia with hyperinflammatory syndrome and acute respiratory failure: a single center study of 100 patients in Brescia, Italy. Autoimmun Rev 2020;19(7):102568. DOI: 10.1016/j.autrev.2020.102568.

6. Guaraldi G, Meschiari M, Cozzi-Lepri A, Milic J, Tonelli R, Menozzi M, et al. Tocilizumab in patients with severe COVID-19: a retrospective cohort study. Lancet Rheumatol 2020;2(8):e474-e484.

7. Price CC, Altice FL, Shyr Y, Koff A, Pischel L, Goshua G, et al. Tocilizumab treatment for cytokine release syndrome in hospitalized COVID19 patients: survival and clinical outcomes. Chest 2020. S00123692(20)31670-6.

8. Cortegiani A, Ippolito M, Greco M, Granone V, Protti A, Gregoretti C, et al. Rationale and evidence on the use of tocilizumab in COVID-19: a systematic review. Pulmonology 2020. DOI: 10.1016/j.pulmoe.202 0.07.003S2531-0437(20)30153-7.

9. Furlow B. COVACTA trial raises questions about tocilizumab's benefit in COVID-19. Lancet Rheumatol 2020;2(10):e592. DOI: 10.1016/S26659913(20)30313-1.

10. Potere N, Di Nisio M, Rizzo G, La Vella M, Polilli E, Agostinone A, et al. Low-dose subcutaneous tocilizumab to prevent disease progression in patients with moderate COVID-19 pneumonia and hyperinflammation. Int J Infect Dis 2020. S1201-9712(20)30616-0 10.1016/j.ijid.2020.07.078.

11. Nishimoto N, Ito K, Takagi N. Safety and efficacy profiles of tocilizumab monotherapy in Japanese patients with rheumatoid arthritis: meta- 
analysis of six initial trials and five long-term extensions. Modern Rheumatol 2010;20(3):222-232. DOI: 10.3109/s10165-010-0279-5.

12. Pettit NN, Nguyen CT, Mutlu GM, Wu D, Kimmig L, Pitrak D, et al. Late onset infectious complications and safety of tocilizumab in the management of COVID-19. J Med Virol 2020. DOI: 10.1002/jmv.26429.
13. Quartuccio L, Sonaglia A, Pecori D, Peghin M, Fabris M, Tascini C, et al. Higher levels of IL-6 early after tocilizumab distinguish survivors from nonsurvivors in COVID-19 pneumonia: a possible indication for deeper targeting of IL-6. J Med Virol 2020. DOI: 10.1002/ jmv.26149. 